\title{
A dual-modality approach of endobiliary radiofrequency ablation and self-expandable metal stent placement to control malignant hemobilia
}
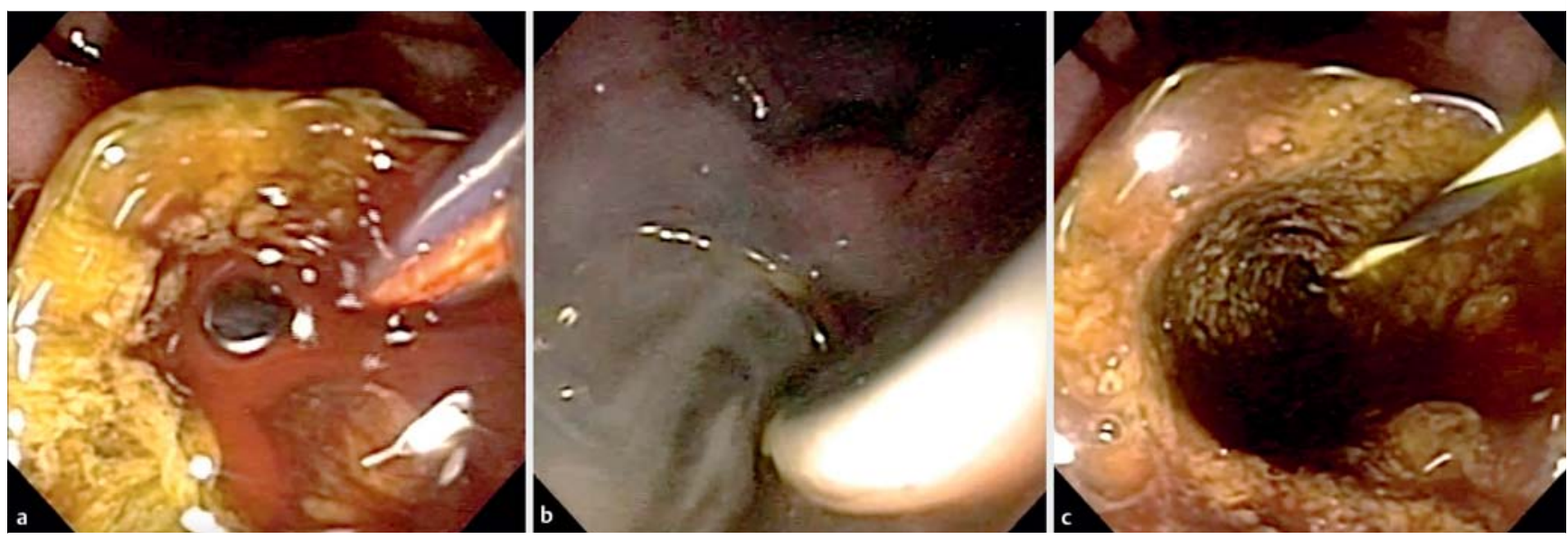

- Fig. 1 Malignant hemobilia in a patient with metastatic pancreatic cancer. a Brisk hemobilia observed during endoscopic retrograde cholangiopancreatography. $\mathbf{b}$ Endobiliary radiofrequency ablation was performed. c Cessation of hemobilia following endobiliary radiofrequency ablation.

A 69-year-old man with a history of metastatic pancreatic cancer involving the head of the pancreas and previous placement of an uncovered self-expandable metal stent (SEMS) for malignant biliary obstruction presented to the hospital with new-onset jaundice, melena, and a drop in hemoglobin from 11.0 to $5.6 \mathrm{~g} / \mathrm{dL}$ over 2 weeks. Given his clinical picture, an urgent upper endoscopy and endoscopic retrograde cholangiopancreatography (ERCP) were performed ( Video 1).

ERCP demonstrated brisk hemobilia with cholangiogram evidence of stent obstruction, which was most likely secondary to tumor ingrowth ( $\triangleright$ Fig. 1 a, - Video 1). Balloon sweeps of the bile duct confirmed suspicion of bleeding from the diffuse tumor ingrowth.

Endobiliary radiofrequency ablation (RFA) was performed for tumor de-

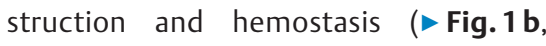
- Video 1). This was chosen as the initial treatment approach because, given the degree of blood loss, it was important to confirm control of bleeding prior to placement of a covered SEMS. The entire length of the stent was ful- gurated with the RFA probe at 10 watts for 90 seconds in sequential fashion. As a result, there was prompt cessation of hemobilia ( $\mathbf{F i g . 1 c} \triangleright$ Video 1). A fully covered SEMS was placed within the uncovered metal stent to prevent continued tumor ingrowth. The patient had no further evidence of bleeding following the intervention, and hemoglobin levels stabilized.

Endoscopic interventions are limited for hemobilia and have historically relied on the tamponade physiology of fully covered SEMS [1-3]. This case presents a

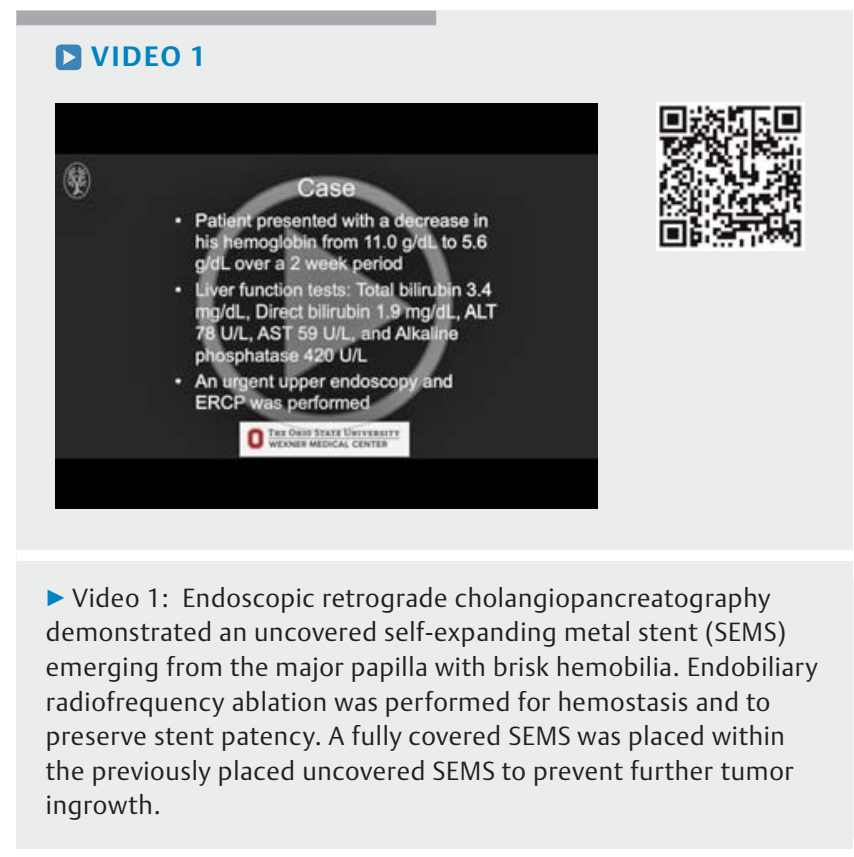


novel technique of using endobiliary RFA to control hemobilia. Recent evidence demonstrates a possible early survival benefit of endobiliary RFA preceding stent placement in patients with metastatic or locally advanced inoperable pancreatic carcinoma, further supporting our management approach [4]. Utilizing a dual-modality approach to controlling malignant hemobilia may result in improved hemostasis and decrease the risk of future bleeding.

Endoscopy_UCTN_Code_TTT_1AR_2AF

Competing interests

None
The Authors

Christopher M. Linz ${ }^{1}$, Rohan M. Modi ${ }^{2}$ Somashekar G. Krishna ${ }^{1}$

1 Division of Gastroenterology, Hepatology, and Nutrition, The Ohio State University Wexner Medical Center, Columbus, Ohio, United States

2 Department of Internal Medicine, The Ohio State University Wexner Medical Center, Columbus, Ohio, United States

\section{Corresponding author}

\section{Somashekar G. Krishna, MD, MPH}

Sections of Pancreatic Disorders and Advanced Endoscopy, Division of Gastroenterology, Hepatology, and Nutrition, 395 W. 12th Avenue, 2nd floor, Columbus, Ohio 43210-1240, United States, Sgkrishna@gmail.com,

somashekar.krishna@osumc.edu

\section{References}

[1] Barresi L, Tarantino I, Ligresti D et al. Fully covered self-expandable metal stent treatment of spurting bleeding into the biliary tract after endoscopic ultrasound-guided fine-needle aspiration of a solid lesion of the pancreatic head. Endoscopy 2015; 47 (Suppl. 01): 1-2

[2] Goenka MK, Harwani Y, Rai V et al. Fully covered self-expandable metal biliary stent for hemobilia caused by portal biliopathy. Gastrointest Endosc 2014; 80: 1175

[3] Aslinia F, Hawkins L, Darwin P et al. Temporary placement of a fully covered metal stent to tamponade bleeding from endoscopic papillary balloon dilation. Gastrointest Endosc 2012; 76: $911-913$

[4] Kallis Y, Phillips N, Steel A et al. Analysis of endoscopic radiofrequency ablation of biliary malignant strictures in pancreatic cancer suggests potential survival benefit. Dig Dis Sci 2015; 60: 3449-3455

\section{Bibliography}

DOI http://dx.doi.org/10.1055/s-0042-120274

Endoscopy 2017; 49: E21-E22

(c) Georg Thieme Verlag KG

Stuttgart · New York

ISSN 0013-726X 